\title{
COVERING PROPERTIES FOR LQUS WITH NESTED BASES
}

\section{A. ANDRIKOPOULOS and J. N. STABAKIS}

(Received 15 August 1997 and in revised form 10 April 2000)

\begin{abstract}
This paper deals with problems on $L Q U$ spaces which have a nested base; among others we give conditions so that a space $\left(X, \tau_{1}, \tau_{2}\right)$ admits an $L Q \mathcal{U} U$ which generates $\tau_{1}$ and $U^{-1} \tau_{2}$, and give necessary and sufficient conditions for a space to be quasi-metrizable, related to concrete covering properties. We also give a Stone's type characterization of pairwise paracompactness for some categories of $L Q U$ spaces with nested bases.
\end{abstract}

2000 Mathematics Subject Classification. Primary 54E15, 54E55, 54E25.

1. Introduction. J. Williams [12] associated a local uniformity with a nested base, to a certain class of regular spaces which fulfil some covering properties. Some years earlier E. Lane [9, Theorem 3.1] gave some similar covering conditions for a pairwise regular bitopological space to define a quasi-metric on the space, but he left in pending a number of relative questions starting with the one referring to the necessity of the conditions. It is our main purpose to reform the Lane's conditions and establish a local quasi-uniformity with a nested base which gives answers to the questions raised by Lane's paper and for which Williams has responded in the uniform case.

The first problem we confront here can be stated as follows: given a bitopological space $\left(X, \tau_{1}, \tau_{2}\right)$, find conditions such that there is a local quasi-uniformity with a nested base which generates the topology $\tau_{1}$ and its dual generates $\tau_{2}$. Theorem 2.5 solves that problem under conditions which may be considered as generalizations of the ones cited in [12, Theorem 2.9] and [9, Theorem 3.1]. The suggestion of necessary and sufficient conditions for a space to be quasi-metrizable of such a form as those which Lane asks for in his paper, is our second point and Theorem 3.1 gives an answer. The assumptions we put there, easily satisfy the Kopperman-Fox's demands [7, Theorem 1.1], an alternative approach to the subject (see Remark 3.2).

Stone's type theorem for the pairwise paracompactness works well for some definitions like, for instance, those introduced in $[4,11]$ whilst it does not for some others, as in $[1,2,6,10]$. The local quasi-uniformity with a nested base which is constructed in Theorem 2.5 assures the pairwise paracompactness.

2. A generalization of William's and Lane's conditions for metrizability and quasimetrizability. Consider a bitopological space $\left(X, \tau_{1}, \tau_{2}\right)$ and a filter of neighbornets on $X$; we call the filter generalized quasi-uniformity (GQU in brief). We also write $L Q U$ for a locally quasi-uniform space and, as always, we symbolize by $\tau(U)$ the topology generated by a quasi-uniformity $u$. The basic result in relation with the quasimetrizability of an $L Q \cup$ space remains the theorem of P. Fletcher and W. F. Lindgren 
[3, Theorem 7.3] and H. P. A. Künzi [8, Theorem 5] which is stated as follows: a space admits a quasi-metric if it admits an $L Q \cup \cup$ with a countable base such that $U^{-1}$ is as well an $L Q$ u.

The following preliminary results are essential.

LEMMA 2.1. Let $\left(X, \tau_{1}, \tau_{2}\right)$ be a bitopological space and consider the class $\mathscr{B}_{1}$ (respectively, $\mathscr{B}_{2}$ ) of $\tau_{2} \times \tau_{1}$-open (respectively, $\tau_{1} \times \tau_{2}$-open) entourages such that for any $\tau_{1}$-neighborhood (respectively, $\tau_{2}$-neighborhood) $M_{1}$ of $x$ (respectively, $M_{2}$ ), there are $\tau_{1}$-neighborhood (respectively, $\tau_{2}$-neighborhood) $N_{1}$ of $x$ (respectively, $N_{2}$ ) and $V_{1} \in \mathscr{B}_{1}$ (respectively, $V_{2} \in \mathscr{B}_{2}$ ) such that $V_{1}\left(N_{1}\right) \subseteq M_{1}$ (respectively, $V_{2}\left(N_{2}\right) \subseteq M_{2}$ ). Then $\mathscr{B}_{1}$ (respectively, $\mathscr{B}_{2}$ ) is a subbase for an $L Q U$ which generates $\tau_{1}$ (respectively, $\tau_{2}$ ).

Proof. We prove the $\tau_{1}$-case only. From the assumptions, $\mathscr{B}_{1}$ is itself a subbase for a $G Q \mathcal{U} \mathscr{V}_{1}$; in fact it is $\tau\left(\mathscr{V}_{1}\right)=\tau_{1}$. Moreover, for any $\tau_{1}$-neighborhood $U[x]$ of $x$, there is another $\tau_{1}$-neighborhood $B$ of $x$ and $V, W$ elements of $\mathscr{B}_{1}$ such that $V[B] \subseteq U[x]$ and $W[x] \subseteq B$. Thus $(W \cap V) \circ(W \cap V)[x] \subseteq V[W[x]] \subseteq V[B] \subseteq U[x]$ and $\mathscr{B}_{1}$ is an $L Q^{\mathcal{U}}$.

LEMMA 2.2. A GQU finer than an LQU and generating the same topology with it, is an LQU as well.

Proof. If $\mathcal{U}$ is the $L Q \mathcal{U}$ and $\mathscr{V}$ the $G Q \mathcal{U}$, then given $x$ and $V \in \mathscr{V}$ there is $U \in \mathscr{U}$ such that $U[x] \subseteq V[x]$, whilst there is another $U^{*} \in \mathcal{U}$, such that $U^{* 2}[x] \subseteq U[x]$. Since $V$ is finer than $U$, there is $V^{*} \in \mathscr{V}$ such that $V^{*} \subseteq U^{*}$, hence $V^{* 2}[x] \subseteq V[x]$.

After J. Williams [12, Definition 2.3], we give the following definition.

DEFINITION 2.3. We call cofinality of a $G Q \mathcal{U}$ the least cardinal $\kappa$ for which the given $G Q \mathcal{U}$ has a base of cardinality $\kappa$.

LEMMA 2.4. If two families $\left\{\mathscr{A}_{\alpha} \mid \alpha \in \kappa\right\}$ and $\left\{\mathscr{P}_{\beta} \mid \beta \in \kappa\right\}$ of subsets have the same cardinality $\kappa$ and are nested, then the family $\left\{\left(A_{\alpha} \times B_{\alpha}\right) \mid \alpha \in \kappa\right\}$ is nested and cofinal to the family $\left\{A_{\alpha} \times B_{\beta} \mid \alpha \in \kappa, \beta \in \kappa\right\}$. (Evidently, given $i \in \kappa$ and $j \in \kappa$, there is an $\alpha \in \kappa$ such that $A_{\alpha} \times B_{\alpha} \subseteq A_{i} \times B_{j}$.)

We now come to one of our basic results. The conditions we have put may be considered as generalizations of the J. William's and E. P. Lane's respective assumptions in [12, Theorem 2.9] and [9, Theorem 3.1].

THEOREM 2.5. Let $\left(X, \tau_{1}, \tau_{2}\right)$ be a bitopological pairwise regular space and $\left\{\mathscr{A}_{\alpha} \mid\right.$ $\alpha \in I\},\left\{\mathscr{B}_{\beta} \mid \beta \in I\right\}$ be nested classes of families of subsets of $X$. For any $\alpha$ and $\beta$ in $I$ and any $\mathscr{A} \subseteq \mathscr{A}_{\alpha}$ and $\mathscr{B} \subseteq \mathscr{B}_{\beta}$, we put

$$
\stackrel{\circ}{A}=\left\{\stackrel{\circ}{A}=\operatorname{int}_{\tau_{1}} A \mid A \in \mathscr{A}\right\}, \quad \stackrel{\circ}{B}=\left\{\stackrel{\circ}{B}=\operatorname{int}_{\tau_{2}} B \mid B \in \mathscr{B}\right\} .
$$

We assume that $(1) \cup_{\alpha} \stackrel{A}{A}$ and $\cup_{\beta} \stackrel{\circ}{9}$ are bases for the topologies $\tau_{1}$ and $\tau_{2}$, respectively. (2) $\cap_{\alpha} \stackrel{\circ}{\AA}$ is $\tau_{1}$-open and $\cap\left\{X \backslash \mathrm{cl}_{\tau_{2}} A \mid A \in \mathscr{A}\right\}$ is $\tau_{2}$-open. (3) $\cap_{\beta} \stackrel{\circ}{\mathscr{S}}$ is $\tau_{2}$-open and $\cap\{X \backslash$ $\left.\mathrm{cl}_{\tau_{1}} A \mid B \in \mathscr{B}\right\}$ is $\tau_{1}$-open.

Then there is an LQ $U$ U with a nested base such that $\tau(\mathcal{U})=\tau_{1}$ and $\tau\left(U^{-1}\right)=\tau_{2}$. 
Proof. Suppose that the collections $\left(\mathscr{A}_{\alpha}\right)_{\alpha}$ and $\left(\mathscr{R}_{\beta}\right)_{\beta}$ contain $X$ and $\varnothing$.

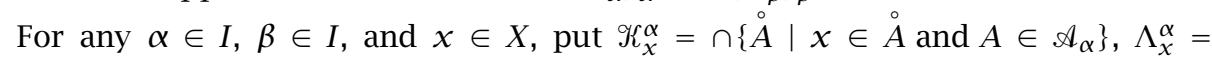
$\cap\left\{X \backslash \mathrm{cl}_{\tau_{2}} A \mid x \in X \backslash \mathrm{cl}_{\tau_{2}} A, A \in \mathscr{A}_{\alpha}\right\} . \mathcal{M}_{x}^{\beta}=\cap\left\{\stackrel{\circ}{B} \mid x \in \stackrel{\circ}{B}\right.$ and $\left.B \in \mathscr{B}_{\beta}\right\}$ and $\mathcal{N}_{x}^{\beta}=$ $\cap\left\{X \backslash \mathrm{cl}_{\tau_{1}} B \mid x \in X \backslash \mathrm{cl}_{\tau_{1}} B, B \in \mathscr{B}_{\beta}\right\}$.

We form $U_{\alpha}=\cup_{x}\left(\Lambda_{x}^{\alpha} \times \mathscr{K}_{x}^{\alpha}\right)$ and $\mathscr{V}_{\beta}=\cup_{x}\left(\mathcal{N}_{x}^{\beta} \times \mu_{x}^{\beta}\right)$ and show that each of the families $\left(\mathcal{U}_{\alpha}\right)_{\alpha}$ and $\left(\mathscr{V}_{\beta}\right)_{\beta}$ forms a nested base for an $L Q \mathcal{U}$ compatible with $\tau_{1}$ and $\tau_{2}$, respectively. We prove it for the first family.

The family $\left\{U_{\alpha} \mid \alpha \in I\right\}$ is nested. In fact, if $\alpha \leq \beta$, then $\Lambda_{x}^{\beta} \subseteq \Lambda_{x}^{\alpha}$ and $K_{x}^{\beta} \subseteq K_{x}^{\alpha}$, hence $u_{\alpha} \subseteq U_{\beta}$. Next, if $x \in X$ and $A \in \cup_{\alpha} \mathscr{A}_{\alpha}$, we can choose $\beta \in I$ and $B \in \mathscr{B}_{\beta}$, such that $x \in B \subseteq \mathrm{cl}_{\tau_{2}} B \subseteq A$. Moreover, if $\gamma \in I, B \in \mathcal{A}_{\gamma}$, and $A \in \mathcal{A}_{\gamma}$, then $u_{\gamma}[B]=\cup_{y}\left\{\mathcal{K}_{y}^{\gamma} \mid\right.$ $\left.\Lambda_{y}^{y} \cap B \neq \varnothing, y \in X\right\}$. If now $y \in A$, then $\Lambda_{y}^{y} \subseteq A$, hence $\cup_{y \in A} \Lambda_{y}^{y} \subseteq A$. If $y \notin A$, then $y \notin \mathrm{cl}_{\tau_{2}} B$ or $y \in X \backslash \mathrm{cl}_{\tau_{2}} B$ and since $\Lambda_{y}^{y} \subseteq X \backslash \mathrm{cl}_{\tau_{2}} B$, it follows that $\Lambda_{y}^{y} \cap B=\varnothing$. Hence $u_{y}[B] \subseteq A$ in any case and from Lemma 2.1 the family $\Gamma=\left\{u_{\alpha} \mid \alpha \in I\right\}$ is a base for an $L Q U$ such that $\tau(\Gamma)=\tau_{1}$.

It also follows that $u_{\alpha}^{-1}[x]=\cup_{y}\left\{\Lambda_{y}^{\alpha}: x \in k_{y}^{\alpha}\right\}$, hence it is $\tau_{2}$-open and $\tau\left(\Gamma^{-1}\right) \subseteq \tau_{2}$. We also have $E=\left\{\mathscr{V}_{\beta} \mid \beta \in \mathscr{I}\right\}$ is an $L Q \mathcal{U}$ such that $\tau(E)=\tau_{2}$ and $\tau\left(E^{-1}\right) \subseteq \tau_{1}$.

Let $F=\Gamma \vee E^{-1}$. Then $\tau(F)=\tau_{1}$, hence by Lemma 2.2, $F$ is an $L Q \mathcal{U}$. We now pick up (after Lemma 2.4) a nested family $\mathcal{U}$ of entourages of the form $\boldsymbol{u}_{\alpha} \cap \mathcal{V}_{\alpha}^{-1}$, where $u_{\alpha}$ and $\mathscr{V}_{\alpha}$ belongs to $\Gamma$ and $E$, respectively. This family induces the topology $\tau_{1}$ as well, thus $\tau(U)=\tau_{1}$. It also follows that $\tau\left(U^{-1}\right)=\tau_{2}$, and the proof is complete.

As always, if we say that bitopological space $\left(X, \tau_{1}, \tau_{2}\right)$ is quasi-metric we mean that there is a quasi-metric $d$ such that the topology induced by $d$ coincides with $\tau_{1}$ and that induced by $d^{-1}$ coincides with $\tau_{2}$.

The following corollaries are directly concluded from Theorem 2.5.

COROLLARY 2.6. If a bitopological pairwise regular space satisfies the assumptions of Theorem 2.5 with the only exception that the given families are countable, then the space is quasi-pseudo-metrizable.

COROLlary 2.7 (see [9, Theorem 3.1]). Let $\left(X, \tau_{1}, \tau_{2}\right)$ be a bitopological space and (preserving the notation of Theorem 2.5) we suppose that the two classes of the fam-

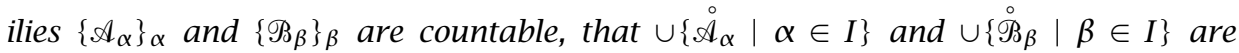
bases for the topologies $\tau_{1}$ and $\tau_{2}$, respectively, and that $\left\{\stackrel{\mathscr{A}}{\alpha}_{\alpha} \mid \alpha \in I\right\}$ and $\left\{\stackrel{\circ}{\beta}_{\beta} \mid\right.$ $\beta \in I\}$ are, both of them, $\tau_{1}$ and $\tau_{2}$-locally finite. Then the space is quasi-pseudometrizable.

An immediate consequence of Theorem 2.5 is the following theorem of J. G. Kelly [5, Theorem 2.5].

THEOREM 2.8. A bitopological pairwise regular space which fulfils the second axiom of countability is quasi-pseudo-metrizable.

In fact, if $\mathscr{P}_{n}$ and $\mathscr{Q}_{n}$ are the countable bases of $\tau_{1}$ and $\tau_{2}$, then the families $\mathscr{A}_{n}=\cup \mathscr{P}_{n}$ and $\mathscr{B}_{n}=\cup \mathscr{2}_{n}$ satisfy the assumptions of Theorem 2.5 and the space is quasi-pseudometrizable. 
The last theorem of this section may be considered as a generalization of the E. P. Lane's theorem [9, Theorem 3.1] with respect to the number of the elements which every family has, in other words, with respect to the cofinality of these families.

THEOREM 2.9. Let $\left(X, \tau_{1}, \tau_{2}\right)$ be a pairwise regular bitopological space, $\left\{\mathscr{A}_{\alpha} \mid \alpha \in I\right\}$ and $\left\{\mathscr{P}_{\beta} \mid \beta \in I\right\}$ be nested collections of $\tau_{1}$ and $\tau_{2}$-open, respectively, families of subsets, each family being $\tau_{1}$ and at the same time $\tau_{2}$ locally finite and $\bigcup\left\{\mathscr{A}_{\alpha} \mid \alpha \in I\right\}$ and $\cup\left\{\stackrel{\circ}{\mathscr{P}}_{\beta} \mid \beta \in I\right\}$ be $\tau_{1}, \tau_{2}$-open bases, respectively. Then the space is quasi-pseudometrizable.

Proof (cf. [12, Proposition 2.10]). From Theorem 2.5 the space admits an $L Q u \cup$ with a nested base such that $U^{-1}$ is also an $L Q U$ with a nested base. If $I=\omega,(\omega$ is the ordinal of the natural numbers), then the result comes from the mentioned FletcherLindgren's theorem. If, on the other hand, $\operatorname{cl}_{\tau_{2}}\{x\}$ and $\mathrm{cl}_{\tau_{1}}\{x\}$ are $\tau_{1}$ and $\tau_{2}$-open, respectively, the space $\left(X, \tau_{1}, \tau_{2}\right)$ admits quasi-pseudo-metrics $d_{1}$ and $d_{2}$ defined as follows:

$$
d_{1}(x, y)=\left\{\begin{array}{ll}
0, & \text { if } y \in \mathrm{cl}_{\tau_{2}}\{x\}, \\
1, & \text { otherwise, }
\end{array} \quad d_{2}(x, y)= \begin{cases}0, & \text { if } y \in \mathrm{cl}_{\tau_{1}}\{x\} \\
1, & \text { otherwise }\end{cases}\right.
$$

From [5, page 86], we deduce that $x \in \mathrm{cl}_{\tau_{2}}\{y\}$ implies $y \in \operatorname{cl}_{\tau_{1}}\{x\}$, hence

$$
d_{1}^{-1}(x, y)=d_{1}(y, x)=\left\{\begin{array}{ll}
0, & \text { if } x \in \mathrm{cl}_{\tau_{2}}\{y\}, \\
1, & \text { otherwise }
\end{array}=\left\{\begin{array}{ll}
0, & \text { if } y \in \mathrm{cl}_{\tau_{1}}\{x\}, \\
1, & \text { otherwise, }
\end{array}=d_{2}(x, y) .\right.\right.
$$

So there remains the case card $I>\omega$ at least one of $\mathrm{cl}_{\tau_{2}}\{x\}, \mathrm{cl}_{\tau_{1}}\{x\}$, say the first, is not $\tau_{1}$-open. We shall derive a contradiction: let $\left\{\mathscr{A}_{\alpha_{n}} \mid n \in \omega\right\}$ be a countable subcollection of $\left\{\mathscr{A}_{\alpha} \mid \alpha \in I\right\}$ such that, for each $n \in \omega, \mathscr{A}_{\alpha_{n}}$ contains a $\tau_{1}$-neighborhood of $x$ and $\mathscr{A}_{a_{n+1}}$ contains a $\tau_{1}$-neighborhood of $x$ which is strictly smaller than any neighborhood of $x$ in $\mathscr{A}_{a_{n}}$. Such an $n$ exists, because any family $\mathscr{A}_{\alpha}(\alpha \in I)$ is $\tau_{1}$-locally finite. Let

$$
N=\bigcap\left\{A \in \mathscr{A}_{a_{n}} \mid x \in A \text { and } n \in \omega\right\},
$$

where $N$ is the countable intersection of open sets and since card $I>\omega, N$ is an open $\tau_{1}$-neighborhood of $x$. Next, we consider a family $\mathscr{A}_{\beta}$ in the collection $\left\{\mathscr{A}_{\alpha} \mid \alpha \in I\right\}$ which contains a $\tau_{1}$-subneighborhood $B$ of $N$. The set $B$ does not belong to any $\mathscr{A}_{\alpha_{n}}$ and we may assume that $\mathscr{A}_{\alpha_{n}} \subset \mathscr{A}_{\beta}$, for any $n \in \omega$. Then, there is an $A \in \mathscr{A}_{a_{n+1}} \backslash \mathscr{A}_{a_{n}}$, where $x \in A$ and $A \in \mathscr{A}_{\beta}$ so that every $\tau_{1}$-neighborhood of $x$ meets infinitely many elements of $\mathscr{A}_{\beta}$, a contradiction.

3. The necessity of Theorem 2.5 assumptions. The theorem which is featured in this section answers the question raised by E. P. Lane [9, page 248], whether there are for a bitopological space sufficient and necessary conditions referring to special coverings, at the end the space to be quasi-metric. We give a solution that slightly changes the conditions of Theorem 2.5 into a more convenient expression.

Let $\left(X, \tau_{1}, \tau_{2}\right)$ be again a bitopological space. We put for any $x \in X$ and for any $\varepsilon>0, B(x, \varepsilon)=\{y \in X \mid d(x, y)<\varepsilon\}, \bar{B}(x, \epsilon)=\{y \in X \mid d(x, y) \leq \epsilon\}$, and $B^{-1}(x, \epsilon)=$ 
$\left\{y \in X \mid d^{-1}(x, y)<\epsilon\right\}$. We also note by $\operatorname{int}_{1} A$ (respectively, $\operatorname{int}_{2} A$ ) the interior with respect to $d$ (respectively, to $d^{-1}$ ) of any $A \subseteq X$. Finally, we recall that a precise refinement (cf. [3, Section 5.4]) of a cover $\mathscr{C}=\left\{C_{\alpha} \mid \alpha \in A\right\}$ of $X$ is a cover $\mathscr{Q}=\left\{R_{\alpha} \mid \alpha \in A\right\}$ provided that for each $\alpha \in A, R_{\alpha} \subseteq C_{\alpha}$.

Before beginning the theorem, some remarks on a $T_{0}$ non $T_{1}$ totally ordered quasipseudo-metrizable space are necessary. Let $(X, d)$ be such a space. If $d(x, y)=0$, then $x \in \operatorname{cl}(y)$ and vice versa. Moreover, if $d(x, y)=0$ and $x \in B(\alpha, \epsilon)$, then $y \in B(\alpha, \epsilon)$ as well, because $d(\alpha, y) \leq d(\alpha, x)+d(x, y)<\epsilon$. The space is always ordered. If we suppose the total ordering and consider the subbase of $] \leftarrow, x]$, as $x$ runs through $X$, $B(\alpha, \epsilon)$ is any sphere and $x$ is larger than any point of $B(\alpha, \varepsilon)$, then any open subset of the form $B\left(x, \epsilon^{\prime}\right), \epsilon^{\prime}>0$, contains $B(\alpha, \epsilon)$, since it contains all $\left.\left.y \in\right] \leftarrow, x\right]$. This means that in this case it is impossible to refine any open covering of $X$ in an effective way, which is a necessary presupposition for the demonstration of a Nagata-Smirnov's-type theorem.

THEOREM 3.1. A $T_{1}$ topological space $\left(X, \tau_{1}, \tau_{2}\right)$ is quasi-pseudo-metrizable if and only if there are two countable collections $\left(\mathscr{A}_{n}\right)_{n \in \omega}$ and $\left(\mathscr{B}_{n}\right)_{n \in \omega}$ of coverings of $X$ consisting of $\tau_{1}$ and $\tau_{2}$-open subsets, respectively, where $\mathscr{A}_{n}=\left\{A_{n i} \mid i \in I\right\}, \mathscr{B}_{n}=\left\{B_{n i} \mid\right.$ $i \in I\}, I$ being the same for all $n$, such that

(1) The family $\cup \mathscr{A}_{n}$ (respectively, $\cup \mathscr{B}_{n}$ ) is a $\tau_{1}$-(respectively, $\tau_{2}$-)open base.

(2) For everyn, $\mathscr{A}_{n+1}$ (respectively, $\mathscr{B}_{n+1}$ ) is precise refinement of $\mathscr{A}_{n}$ (respectively, $\mathscr{B}_{n}$ ).

(3) For any $n$ and any $J \subset I, \cap\left\{A_{n j} \mid j \in J\right\}$ (respectively, $\cap\left\{B_{n j} \mid j \in J\right\}$ ) is a $\tau_{1}$ (respectively, $\tau_{2}{ }^{-}$) neighborhood of $\cap\left\{A_{(n+1) j} \mid j \in J\right\}$ (respectively, $\cap\left\{B_{(n+1) j} \mid j \in J\right\}$ ).

(4) Similarly, $\cap\left\{X \backslash A_{(n+1) j} \mid j \in J\right\}$ (respectively, $\cap\left\{X \backslash B_{(n+1) j} \mid j \in J\right\}$ ) is a $\tau_{2}$ (respectively, $\tau_{1}^{-}$) neighborhood of $\cap\left\{X \backslash A_{n j} \mid j \in J\right\}$ (respectively, $\cap\left\{X \backslash B_{n j} \mid j \in J\right\}$ ).

Proof. For the sufficiency of the statement we follow the demonstration of Theorem 2.5: we construct an $L Q U U$ such that $\tau(\mathcal{U})=\tau_{1}$ (the construction of a $\mathscr{V}$ such that $\tau(\mathscr{V})=\tau_{2}$ is similar), and we arrive, just as in Theorem 2.5, at an $L Q \mathcal{U} \mathscr{W}$ such that $\tau(\mathcal{W})=\tau_{1}$ and $\tau\left(\mathcal{W}^{-1}\right)=\tau_{2}$, as desired. We only define $U$ : for any $x$ and for any $n \in \omega$ put $\mathscr{K}_{x}^{n}=\operatorname{int}_{1}\left\{\cap A_{n i} \mid x \in \cap A_{(n+1) i}, i \in I\right\}, \Lambda_{x}^{n}=\operatorname{int}_{2}\left\{\cap\left(X \backslash A_{(n+2) i}\right) \mid x \in\right.$ $\left.\cap\left(X \backslash A_{(n+1) i}\right), i \in I\right\}$.

Then the family $u=\left\{U_{n} \mid n \in \omega\right\}$, where $U_{n}=\cup\left\{\Lambda_{x}^{n} \times \mathscr{K}_{x}^{n} \mid x \in X\right\}$ is a base for an $L Q U$ compatible with $\tau_{1}$. More precisely, we show that for any $A \in \cup \mathscr{A}_{n}$, there is another member $B$ of the family and a $U_{m} \in \mathcal{U}$ such that $U_{m}[B] \subseteq A$. In fact, given $A=A_{m i}$ there are $B^{*}=A_{(m+1) i}$ and $B=A_{(m+2) i}$ such that $B \subseteq B^{*} \subseteq A$. Then $U_{m}[B]=$ $\cup\left\{\mathscr{K}_{x}^{m} \mid \Lambda_{x}^{m} \cap B \neq \varnothing, x \in X\right\}$. So, if $x \in B^{*}, \mathscr{K}_{x}^{m} \subseteq \cap\left\{A_{m i} \mid i \in I\right\} \subseteq A$. If $x \notin B^{*}$, then $x \in X \backslash B^{*}$, and since $X \backslash B \in\left\{\left(X \backslash A_{(m+2) i}\right) \mid i \in I\right\}$, we have $\Lambda_{x}^{m}=\operatorname{int}_{2}\left\{\cap\left(X \backslash A_{(m+2) i}\right) \mid\right.$ $i \in I\} \subseteq X \backslash B$, hence $\Lambda_{x}^{m} \cap B=\varnothing$. Thus $U_{m}[B] \subseteq A$.

We prove the necessity for the family $\left(\mathscr{A}_{n}\right)_{n \in \omega}$. We suppose that there is a quasipseudo-metric $d$ such that $\tau(d)=\tau_{1}$ and $\tau\left(d^{-1}\right)=\tau_{2}$.

Let $\mathscr{2}^{(m)}=\{B(x, 1 / m) \mid x \in X, m \in \omega\}$ be a covering of $X$. Put $S_{n}[B(x, 1 / m)]=\{t \in$ $X \mid B(t, 1 / n) \subseteq B(x, 1 / m)\}$ and $E_{n}[B(x, 1 / m)]=\cup\left\{B(x, 1 / 3 m) \mid x \in S_{n}[B(x, 1 / m)]\right\}$. Remark that if $m>n$, then $E_{n}[B(x, 1 / m)]=\varnothing$. We suppose that $n \geq m$.

We prove that a subfamily of the family $\left\{E_{n}[B(x, 1 / m)] \mid m \in \omega, n \in \omega\right\}$, covering of $X$, fulfils the statements (2) and (3). (We have put $\bar{B}$ instead of $\operatorname{cl} B$.) 
There holds:

$$
B\left(x, \frac{1}{m(m+1)}\right) \subseteq \bar{B}\left(x, \frac{1}{m(m+1)}\right) \subseteq S_{m+1}\left[B\left(x, \frac{1}{m}\right)\right] \subseteq E_{m+1}\left[B\left(x, \frac{1}{m}\right)\right] .
$$

In fact, if $t \in \bar{B}(x, 1 / m(m+1))$ and $\lambda \in B(t, 1 /(m+1))$, then $d(x, \lambda) \leq d(x, t)+$ $d(t, \lambda)<1 / m$, hence $B(t, 1 /(m+1)) \subseteq B(x, 1 / m)$ and $t \in S_{m+1}[B(x, 1 / m)]$. We also have $E_{m(m+1)+1}[B(x, 1 / m(m+1))] \subseteq B(x, 1 / m(m+1)) \subseteq \bar{B}(x, 1 / m(m+1)) \subseteq E_{m+1}$ $[B(x, 1 / m)]$. The latter means that the covering $\left\{E_{m(m+1)+1}[B(x, 1 / m(m+1))] \mid x \in\right.$ $X\}$ is a precise refinement of $\left\{E_{m+1}[B(x, 1 / m)] \mid x \in X\right\}$. On the other hand, if $t \in$ $\cap_{x} E_{m(m+1)+1}[B(x, 1 / m(m+1))]$, then $t \in S_{m+1}[B(x, 1 / m)]$ and thus $B(t, 1 / 3(m+$ $1)) \subseteq E_{m+1}[B(x, 1 / m)]$, or $B(t, 1 / 3(m+1)) \subseteq \cap_{x} E_{m+1}[B(x, 1 / m)]$ and (3) has been proved.

We now consider $t \in \cap_{x}\left\{X \backslash E_{m+1}[B(x, 1 / m)]\right\}$. If $\kappa \in S_{m+1}[B(x, 1 / m)], d(\kappa, t)>$ $1 / 3(m+1)$ and since $E_{m(m+1)+1}[B(x, 1 / m(m+1))] \subseteq S_{m+1}[B(x, 1 / m)]$, for any $\lambda \in$ $E_{m(m+1)+1}[B(x, 1 / m(m+1))]$, it follows that $d(\lambda, t)>1 / m(m+1)$, hence

$$
E_{m(m+1)+1}\left[B\left(x, \frac{1}{m(m+1)}\right)\right] \bigcap B^{-1}\left(t, \frac{1}{3(m+1)}\right)=\varnothing .
$$

Thus $B^{-1}(t, 1 / 3(m+1)) \subseteq \cap_{x}\left\{X \backslash E_{m(m+1)+1}[B(x, 1 / m(m+1))]\right\}$ and the statement (4) has been proved.

The required family $\left(\mathscr{A}_{n}\right)_{n \in \omega}$ is defined as follows:

$$
\mathscr{E}_{n+1}=\left\{E_{n+1}\left[B\left(x, \frac{1}{n}\right)\right] \mid x \in X, n \in \omega \backslash\{0\}\right\} .
$$

Define $\mathscr{A}_{2}=\mathscr{E}_{2}$ and if $\mathscr{A}_{n}=\mathscr{E}_{n^{*}}, n, n^{*}$ in $\omega$, then $\mathscr{A}_{n+1}=\mathscr{E}_{n *\left(n^{*+1}\right)}$ and the proof is complete.

REMARK 3.2. It is evident that for the above-mentioned pairs $\left(\mathscr{A}_{n}, \mathscr{A}_{n+1}\right), \mathscr{A}_{n+1}$ are $\tau_{1}$-cocushioned, $\tau_{2}$-cushioned of $\mathscr{A}_{n}$ and for the pairs $\left(\mathscr{B}_{n}, \mathscr{B}_{n+1}\right), \mathscr{B}_{n+1}$ are $\tau_{2}$ cocushioned, $\tau_{1}$-cushioned of $\mathscr{B}_{n}$ as well.

In fact, it follows that (i) $\cap\left(X \backslash A_{n i}\right) \subseteq \operatorname{int}_{\tau_{2}} \cap\left(X \backslash A_{(n+1) i}\right)$ or $X \backslash$ int $_{\tau_{2}} \cap\left(X \backslash A_{(n+1) i}\right) \subseteq$ $X \backslash \cap\left(X \backslash A_{n i}\right)=\cup A_{n i}$. On the other hand, (ii) $\mathrm{cl}_{\tau_{2}} \cup\left(A_{(n+1) i}\right) \subseteq X \backslash$ int $_{\tau_{2}} \cap\left(X \backslash A_{(n+1) i}\right)$.

In fact, if $t \in \operatorname{int}_{\tau_{2}} \cap\left(X \backslash A_{(n+1) i}\right)$, then there is a $\tau_{2}$-neighborhood $V_{t}^{\tau_{2}}$ of $t$ such that $V_{t}^{\tau_{2}} \subseteq X \backslash A_{(n+1) i}$ for any $i$ or $V_{t}^{\tau_{2}} \cap A_{(n+1) i}=\varnothing$. Thus $V_{t}^{\tau_{2}} \cap\left(\cup A_{(n+1) i}\right)=\varnothing$ or $t \notin \mathrm{cl}_{\tau_{2}}\left(\cup A_{(n+1) i}\right)$ or $t \in X \backslash \mathrm{cl}_{\tau_{2}}\left(\cup A_{(n+1) i}\right)$. Thus $\mathrm{cl}_{\mathrm{T}_{2}}\left(\cup A_{(n+1) i}\right) \subseteq A_{n i}$. Thus the assumptions of Kopperman-Fox's theorem [7, Theorem 1.1] are fulfilled and at the same time give an answer to R. D. Kopperman's question [7, page 106, Question c].

\section{Some consequences of Theorem 2.5 .}

The PAIRWISE PARACOMPACTNESS. Since a metrizable space is paracompact, it is a reasonable requirement for a quasi-metrizable space to be pairwise paracompact with respect to any definition of the pairwise paracompactness. Nevertheless, among the relative definitions in M. C. Datta [1], P. Fletcher [2], C. Konstadilaki-Savopoulou and I. L. Reilly [6], T. G. Raghavan [10], S. Romaguera and J. Marín [11] and M. Ganster 
and I. L. Reilly [4], only the last two satisfy this demand, although all of them coincide with the "paracompactness" in the case where the bitopological spaces are reduced to simple ones. Furthermore, J. Williams [12, Theorem 2.8] demonstrated that locally uniform spaces with nested bases are paracompact. We show that, according to the definitions introduced in $[4,11]$, the pairwise paracompactness is directly derived from quasi-uniformities with a nested base. We will symbolize in the text: [11]- or [4]-pairwise paracompactness, respectively.

For our convenience, we shortly refer to some definitions (cf. mainly in S. Romaguera [11, page 236]).

JUNNILA'S DEFINITION OF PARACOMPACTNESS. A regular space $X$ is paracompact if and only if, given a cover $\mathscr{F}$ of $X$, there is for any $x$ a sequence $\left\{U_{n}[x]: n \in \omega\right\}$ of neighborhoods of $x$ such that (i) $y \in U_{n}[x] \Leftrightarrow x \in U_{n}[y]$, and (ii) if $x \in X$, there is $n \in \omega$ and $G \in \mathscr{F}$ such that $U_{n}^{2}[x] \subseteq G$.

By a pair open cover of a bitopological space $(X, \mathscr{P}, 2)$ we mean a family of pairs $\left\{\left(G_{\alpha}, H_{\alpha}\right) \mid \alpha \in I\right\}$ such that $G_{\alpha}$ is $\mathscr{P}$-open, $H_{\alpha}$ 2-open, (ii) $\mathscr{G}=\left\{G_{\alpha}: \alpha \in I\right\}$ and $\mathscr{H}=$ $\left\{H_{\alpha}: \alpha \in I\right\}$ are covers of $X$ and (iii) for each $x \in X$ there is an $\alpha \in I$ such that $x \in G_{\alpha} \cap H_{\alpha}$.

THE [11]-PAIRWISE PARACOMPACTNESS. A pairwise regular space $(X, \mathscr{P}, 2)$ is pairwise paracompact if and only if given a pair cover $(\mathscr{G}, \mathscr{H})$, there is for every $x$ a sequence $\left\{U_{n}[x]: n \in \mathbb{N}\right\}$ of $\mathscr{P}$-neighborhoods and a sequence $\left\{V_{n}[x]: n \in \mathbb{N}\right\}$ of 2 neighborhoods of $x$ such that (i) $y \in U_{n}[x] \Leftrightarrow x \in V_{n}[y]$, (ii) for that $x$, there is an $n \in \omega$ and a pair $\left(G_{\alpha}, H_{\alpha}\right)$ in $(\mathscr{G}, \mathscr{H})$ such that $U_{n}^{2}[x] \subseteq G_{\alpha}$ and $V_{n}^{2}[x] \subseteq H_{\alpha}$.

The [4]-PAIRWISE PARACOMPACTNESS. A pairwise regular space $(X, \mathscr{P}, \mathscr{2})$ is $\delta$ pairwise paracompact if every $\mathscr{P}$ or 2 -open cover of $X$ has a $\mathscr{P} \vee \mathscr{Q}$ (the supremum of $\mathscr{P}$ and 2 ) locally finite refinement.

We firstly give (Theorems 4.4, 4.5, and 4.6) conditions under which we may construct on a space quasi-uniformities with nested bases.

DEFINITION 4.1. A quasi-uniformity $(X, \mathcal{U})$ enjoys the neighborhood property if for any $x \in X$ and any $U \in \mathcal{U}$, there is a $V_{x} \in \mathcal{U}$ such that $V_{x}^{-1}[x] \times V_{x}[x] \subseteq U$.

Proposition 4.2. If in $\left(X, u, u^{-1}\right), \mathcal{U}$ and $u^{-1}$ are $L Q U s$, then $u^{2}$ fulfils the neighborhood property $\left(U^{2}=\left\{U \in \mathcal{U} \mid(\exists V \in \mathcal{U})\left[V^{2} \subset U\right]\right\}\right)$.

Proof. Let $x \in X$ and $U \in \mathcal{U}^{2}$. Then, there are $W \in \mathcal{U}$ such that $W^{2} \subseteq U$ and $V_{1 x}, V_{2 x}$ in $U$ such that $V_{1 x}^{2}[x] \subseteq W[x]$ and $V_{2 x}^{-2}[x] \subseteq W^{-1}[x]$. Put $V_{x}=V_{1 x} \cap V_{2 x}$, then $V_{x}^{-2}[x] \times V_{x}^{2}[x] \subseteq W^{-1}(x) \times W(x) \subseteq W^{2} \subseteq U$ and $V_{x}^{2} \in U^{2}$.

Proposition 4.3. If $U$ is an $L Q U$, then $U^{2}$ is also an $L Q U$, which generates the same topology as $U$.

Proof. Given $V \in \mathcal{U}$ and $x \in X$, there is a $W_{x} \in \mathcal{U}$ such that $W_{x}^{2}[x] \subseteq V[x], W_{x}^{2} \in$ $U^{2}$, hence $\tau(u) \subseteq \tau\left(u^{2}\right)$. If, on the other hand, $W \in U^{2}$ and $x \in X$, then there is a $V \in \mathcal{U}$ such that $V^{2} \subseteq W$, hence $V[x] \subseteq V^{2}[x] \subseteq W[x]$ and $\tau\left(u^{2}\right) \subseteq \tau(u)$. Moreover, evidently, $U^{2}$ is $L Q U$. 
THEOREM 4.4. If $U$ and $U^{-1}$ are LQUs on $X$ with nested bases, then the set of the diagonal neighborhoods generates a quasi-uniform topology equivalent to $\tau(\mathcal{U})$.

Proof. After Propositions 4.2 and 4.3, we may suppose that $u$ is an $L Q U$ with a nested base $\mathscr{A}$ which has the neighborhood property. Let $U$ be a neighborhood of the diagonal and $\mathscr{B}=\{U \cap V: V \in \mathscr{A}\} . \mathscr{B}$ is a nested class of neighborhoods of the diagonal which generates a $G Q U$ finer than $U$. Hence, by Lemma 2.2, $\mathscr{B}$ is a base for a quasi-uniformity $\mathcal{W}$; furthermore, $\mathcal{W}$ fulfils the neighborhood property and induces on $X$ a topology equivalent to that induced by $U$.

Since $U \in \mathcal{W}$, it implies that for any $x \in X$ there is a $V_{x} \in \mathcal{W}$ such that $V_{x}^{-1}[x] \times$ $V_{x}[x] \subseteq U$, and a $W \in \mathscr{W}$ such that $W^{-3}[x] \subseteq V_{x}^{-1}[x]$ and $W^{3}[x] \subseteq V_{x}[x]$, hence $W_{x}^{-3}[x] \times W_{x}^{3}[x] \subseteq V_{x}^{-1} \times V_{x}[x] \subseteq U$.

Put

$$
W=\bigcup\left\{W_{x}^{-1}[x] \times W_{x}[x] \mid x \in X\right\},
$$

where $W$ is a neighborhood of the diagonal. We will show that $W \circ W \subseteq U$.

Let $(x, y) \in W \circ W$, there is $z$ such that $(x, z) \in W,(z, y) \in W$, consequently there are $\alpha, \beta$ in $X$ such that $x \in W_{\alpha}^{-1}[\alpha], z \in W_{\alpha}[\alpha]$ and $z \in W_{\beta}^{-1}[\beta], y \in W_{\beta}[\beta]$. If $W_{\alpha} \subseteq W_{\beta}$, then $x \in W_{\alpha}^{-2} \circ W_{\beta}^{-1}[\beta] \subseteq W_{\beta}^{-3}[\beta]$ and $y \in W_{\beta}[\beta]$, hence $(x, y) \in W_{\beta}^{-3}[\beta] \times W_{\beta}[\beta] \subseteq U$. If $W_{\beta} \subseteq W_{\alpha}$, then $y \in W_{\beta}^{2} \circ W_{\alpha}[\alpha] \subseteq W_{\alpha}^{3}[\alpha]$, hence $(x, y) \in W_{\alpha}^{-1}[\alpha] \times W_{\alpha}^{3}[\alpha] \subseteq U$, or $W \circ W \subseteq U$.

THEOREM 4.5. If in a bitopological space $\left(X, \tau_{1}, \tau_{2}\right)$, where both topologies induce quasi-uniformities with nested bases which have the same cofinality $\mathrm{N}$, a family A of $\tau_{2} \times \tau_{1}$-neighborhoods of the diagonal has cardinality less than $\mathrm{N}$, then $\cap \mathcal{A}$ is a neighborhood of the diagonal.

Proof. It is known (cf. [12, Theorem 2.4]) that in a uniform (as well as in a quasiuniform) space with a nested base of cofinality $\mathrm{k}$, any collection of open sets and of cardinality less than $\mathrm{N}$, has as intersection an open set. If $\mathscr{A}$ is the collection and $x \in X$, then for any $A \in \mathscr{A}$, there are $K_{A}[x]$ and $\Lambda_{A}[x], \tau_{1}$ and $\tau_{2}$-neighborhoods of $x$, such that $\Lambda_{A}[x] \times K_{A}[x] \subseteq A$. If $K=\cap\left\{K_{A}[x]: A \in \mathscr{A}\right\}$ and $\Lambda=\cap\left\{\Lambda_{A}: A \in \mathscr{A}\right\}$, then from the above statement and the fact that $K$ and $\Lambda$ are $\tau_{1}$ and $\tau_{2}$-neighborhoods of $x$, it implies that $\Lambda \times K \subseteq \cap A$. Hence $\cap \mathscr{A}$ is a $\tau_{2} \times \tau_{1}$-neighborhood of the diagonal.

THEOREM 4.6. If $u$ and $u^{-1}$ are LQ $u s$ with nested bases and are of the same cofinality, then there is a quasi-uniformity with a nested base which generates the same, as the $U$, topology and has the same cofinality.

Proof. If $\mathrm{N}$ is the common cofinality of $U$ and $U^{-1}$, and $W_{\lambda}(\lambda \in \kappa)$ is any neighborhood of the diagonal, then by Theorem 4.4 there is a neighborhood $U_{\lambda+1}$ of the diagonal such that $U_{\lambda+1} \circ U_{\lambda+1} \subseteq U_{\lambda}$. If $\lambda$ is a limit ordinal number less than $\mathrm{N}$ and each $U_{\alpha}$, for $\alpha<\lambda$, has been chosen, then put $U_{\lambda}=\cap\left\{U_{\alpha}: \alpha<\alpha\right\}, U_{\lambda}$ is by Theorem 4.5 a neighborhood of the diagonal. The rest are trivial.

We come now to discuss the $[4,11]$-pairwise paracompactness. As usual we denote by $U^{*}$ the uniformity which is the supremum of the quasi-uniformities $u$ and $u^{-1}$ defined on $X$. 
THEOREM 4.7. If for a bitopological space $\left(X, \tau_{1}, \tau_{2}\right)$ there are LQ $u s$ u and $u^{-1}$ with a nested base (both of them) and $\tau(U)=\tau_{1}, \tau\left(U^{-1}\right)=\tau_{2}$, then the space is $\delta$-pairwise paracompact.

Proof. After Theorem 4.6, there is a quasi-uniformity $\mathcal{W}$ whose dual $\mathscr{W}^{-1}$ is also quasi-uniformity, both of them have nested bases, they may be extended (Theorem 4.6) until they reach the same cofinality $\mathrm{s}$ and, finally, they generate topologies on $X$ equivalent to $\tau(U)$ and $\tau\left(U^{-1}\right)$, respectively. We may also assume that the uniformity $\mathscr{W}^{*}$ has a nested base with cofinality $\mathrm{x}$.

If $\mathrm{N}=\omega$, the space $(X, \mathcal{U})$ is quasi-metrizable, hence the bitopological space $\left(X, \tau_{1}, \tau_{2}\right)$ is $\delta$-pairwise paracompact.

Let $\alpha>\omega$. From a E. Zakon's result [13, Theorem 2.1] we may consider that the nested base of the uniformity $\mathcal{W}^{*}$ consists of equivalent relations, which leads-after [12, Theorem 2.7]-to the fact that every $\tau_{1} \vee \tau_{2}=\tau\left(\mathscr{W}^{*}\right)$-open cover $\mathscr{A}$ there is a $\tau\left(W^{*}\right)$-discrete refinement such that there is a partition, say $\left\{S_{\alpha_{x}}[x] \mid x \in X\right\}$, of the space, where $\left\{S_{\alpha_{x}}[x] \mid x \in X\right\}$ refines $\mathscr{A}$. Thus, the $\tau_{1} \vee \tau_{2}$-opening covering $\mathscr{A}$ has a locally finite refinement and the $\delta$-pairwise paracompactness has been proved.

THEOREM 4.8. If for a bitopological space $\left(X, \tau_{1}, \tau_{2}\right)$ there are LQ $u s$ und $u^{-1}$ with a nested base (both of them) and $\tau(U)=\tau_{1}, \tau\left(U^{-1}\right)=\tau_{2}$, then the space is [11]-pairwise paracompact.

Proof. Let $\left(X, \tau_{1}, \tau_{2}\right), \mathscr{W}$ and $\mathscr{W}^{-1}$ be as in Theorem 4.7 . If the cofinality of the base equals $\omega$, the space is quasi-metrizable, hence [11]-pairwise paracompact.

Let the cofinality of $\kappa$ be larger than $\omega$ and $(\mathscr{G}, \mathscr{H})$ be a $\left(\tau(\mathcal{W}), \tau\left(\mathcal{W}^{-1}\right)\right)$-open pair cover of $X$, hence $\mathscr{G} \cap \mathcal{H}=\{G \cap H \mid G \in \mathscr{G}, H \in \mathscr{H}\}$ is a $\tau\left({ }^{*} W^{*}\right)$-cover of $X$. That cover (as we have referred in Theorem 4.7) has a discrete refinement, say $\left\{S_{\alpha_{x}}[x] \mid x \in X\right\}$. Let $S_{\alpha_{x}}[x]$ be the only $\tau\left(W^{*}\right)$-neighborhood of $x$ with respect to this refinement. If $\left\{W_{\alpha}^{*} \mid\right.$ $\kappa \in I\}$ and $\left\{W_{\mathcal{N}} \mid \kappa \in I\right\}$ are $\tau\left({ }^{\mathscr{O}} W^{*}\right)$ - and $\tau\left({ }^{\mathcal{W}}\right)$-bases, respectively, then for any $x \in X$ there is an $\alpha \in I$ such that $W_{\alpha}^{*}=W_{\alpha}[x] \cap W_{\alpha}^{-1}[x] \subseteq S_{\alpha_{x}}[x]$; furthermore if $x \in G_{\alpha} \cap F_{\alpha}$, where $G_{\alpha} \in \mathscr{G}_{\text {and }} H_{\alpha} \in \mathscr{H}$ and we preserve for our convenience the same $\alpha$, then there is an $\alpha^{*}$ such that $W_{\alpha^{*}}^{2}[x] \subseteq W_{\alpha^{*}}^{-1}[x] \subseteq G_{\alpha}$ and $W_{\alpha^{*}}^{-2}[x] \subseteq W_{\alpha^{*}}^{-1}[x] \subseteq H_{\alpha}$ and we may suppose that $W_{\alpha^{*}}[x] \cap W_{\alpha^{*}}^{-1}[x] \subseteq S_{\alpha_{x}}[x] \subseteq G_{\alpha} \cap H_{\alpha}$. On the other hand, we may put $W_{\alpha^{*}}[x]=V_{n}[x]$ and $W_{\alpha^{*}}^{-1}[x]=U_{n}[x]$ for every $n \in \omega$. These two sequences fulfill the requirements of the S. Romaguera [11]-definition of pairwise paracompactness and the proof is complete.

REMARK 4.9. (1) After Theorems 4.7 and 4.8 it is evident that every bitopological space which satisfies the assumptions of Theorem 2.5 is $\delta$-pairwise paracompact as well as [11]-pairwise paracompact.

(2) The quasi-metrizability is equivalent (according to S. Romaguera and J. Marín [11, Theorem 1]) to the facts of being the space [11]-pairwise paracompact plus of being pairwise developable. The latter property is evident under the assumptions of Theorem 2.5. On the other hand, it is worth seeing that in [11] the authors are not concerned with the case of the cofinality being larger than $\omega$, that is, with the case of the space not being quasi-metrizable. In fact, the pairwise development demands the existence of a sequence of pair open covers of the space. 


\section{REFERENCES}

[1] M. C. Datta, Paracompactness in bitopological spaces and an application to quasimetric spaces, Indian J. Pure Appl. Math. 8 (1977), no. 6, 685-690. MR 57\#4094. Zbl 372.54015 .

[2] P. Fletcher, H. B. Hoyle, III, and C. W. Patty, The comparison of topologies, Duke Math. J. 36 (1969), 325-331. MR 39\#3441. Zbl 179.51004.

[3] P. Fletcher and W. F. Lindgren, Quasi-uniform Spaces, Lecture Notes in Pure and Applied Mathematics, vol. 77, Marcel Dekker, New York, 1982. MR 84h:54026. Zbl 501.54018 .

[4] M. Ganster and I. L. Reilly, On pairwise paracompactness, J. Austral. Math. Soc. Ser. A 53 (1992), no. 2, 281-285. MR 93h:54016. Zbl 776.54021.

[5] J. C. Kelly, Bitopological spaces, Proc. London Math. Soc. (3) 13 (1963), 71-89. MR 26\#729. Zbl 107.16401.

[6] C. Konstadilaki-Savopoulou and I. L. Reilly, On Datta's bitopological paracompactness, Indian J. Pure Appl. Math. 12 (1981), no. 7, 799-803. MR 82i:54051. Zbl 468.54023.

[7] R. D. Kopperman, Which topologies are quasimetrizable?, Topology Appl. 52 (1993), no. 2, 99-107. MR 95c:54048. Zbl 801.54026.

[8] H.-P. A. Künzi, On strongly quasimetrizable spaces, Arch. Math. (Basel) 41 (1983), no. 1, 57-63. MR 85f:54058.

[9] E. P. Lane, Bitopological spaces and quasi-uniform spaces, Proc. London Math. Soc. (3) 17 (1967), 241-256. MR 34\#5054. Zbl 152.21101.

[10] T. G. Raghavan and I. L. Reilly, Metrizability of quasi-metric spaces, J. London Math. Soc. (2) 15 (1977), no. 1, 169-172. MR 55\#9042. Zbl 348.54025.

[11] S. Romaguera and J. Marín, On the bitopological extension of the Bing metrization theorem, J. Austral. Math. Soc. Ser. A 44 (1988), no. 2, 233-241. MR 89g:54070. Zbl 648.54027.

[12] J. Williams, Locally uniform spaces, Trans. Amer. Math. Soc. 168 (1972), 435-469. MR 45\#5950. Zbl 235.54026.

[13] E. Zakon, On uniform spaces with quasi-nested base, Trans. Amer. Math. Soc. 133 (1968), 373-384. MR 37\#3514. Zbl 181.25804.

A. ANDRikopoulos: Department of MAthematics, University of PATras, 26500 PATRAS, GREECE

J. N. Stabakis: Department of Mathematics, University of PAtras, 26500 Patras, GREECE

E-mail address: jns@math. upatras.gr 


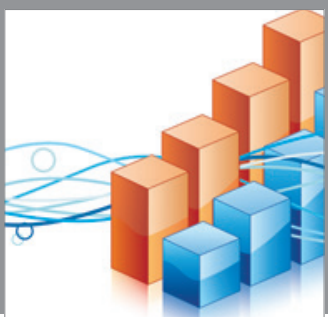

Advances in

Operations Research

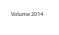

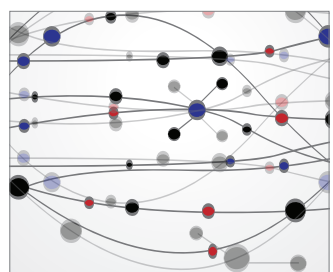

\section{The Scientific} World Journal
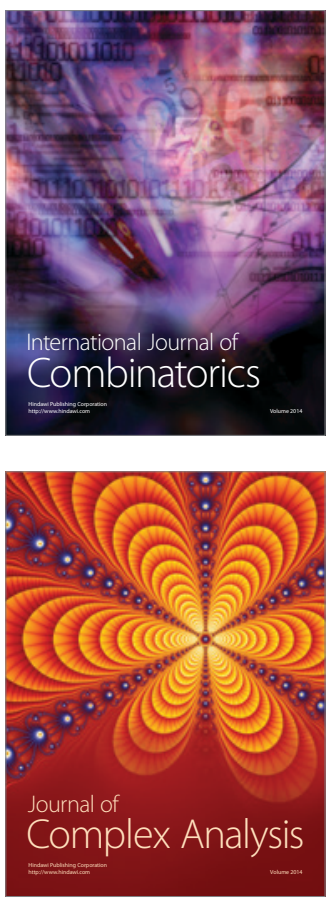

International Journal of

Mathematics and

Mathematical

Sciences
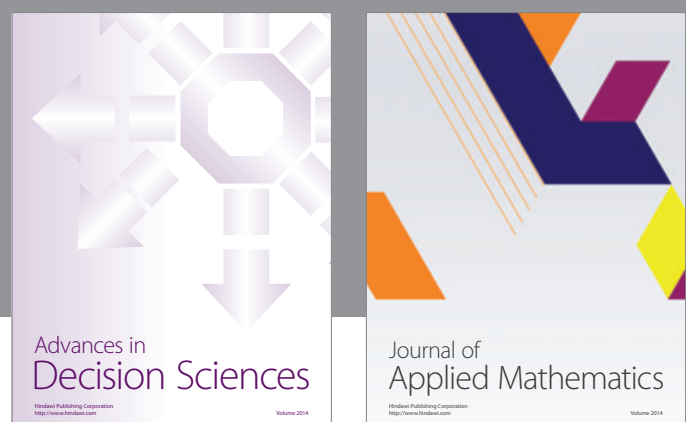

Journal of

Applied Mathematics
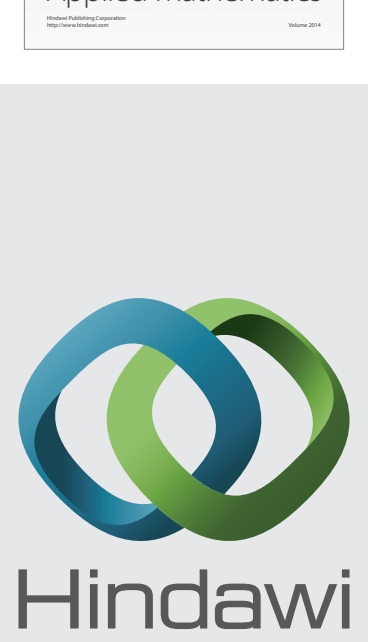

Submit your manuscripts at http://www.hindawi.com
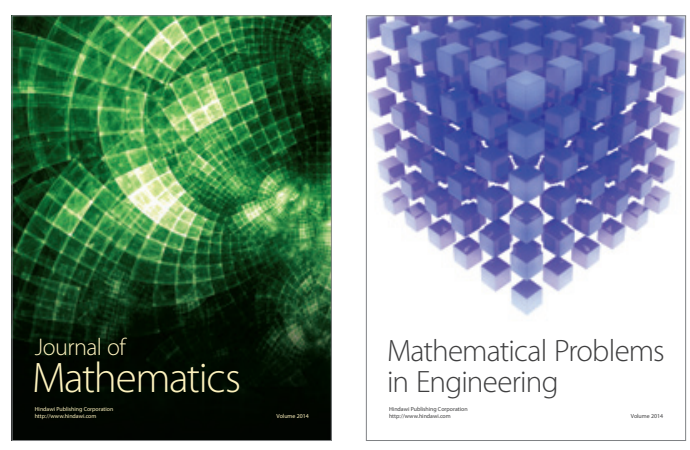

Mathematical Problems in Engineering
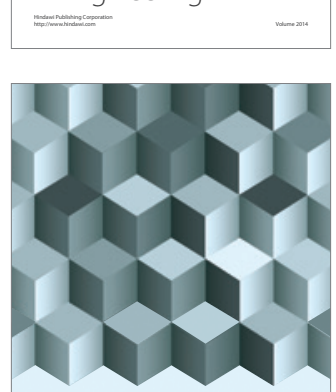

Journal of

Function Spaces
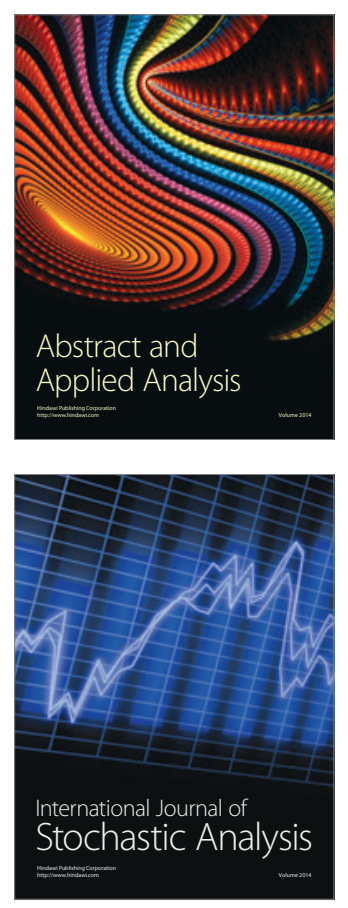

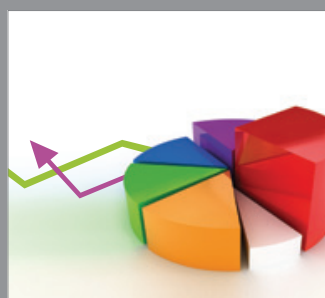

ournal of

Probability and Statistics

Promensencen
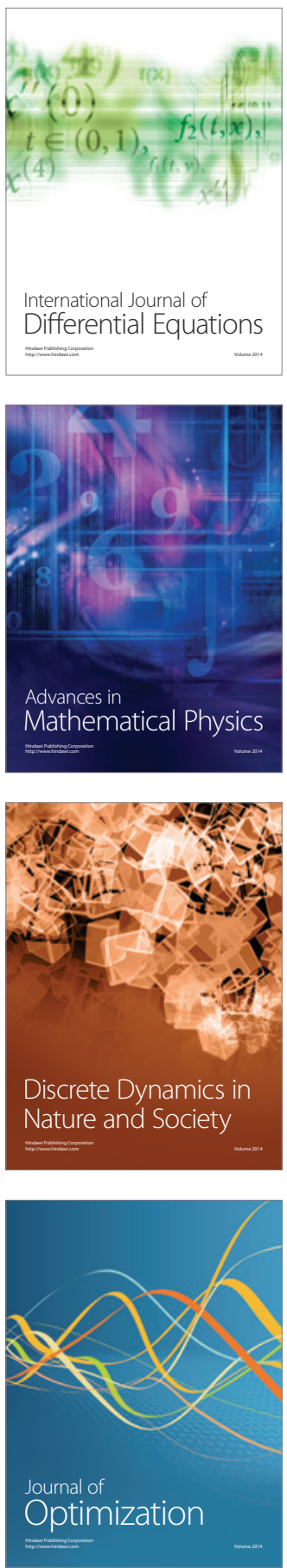